\title{
Carcinoma del uraco: una extraña entidad
}

\section{Urachal Carcinoma: a Strange Entity}

\author{
Maria Esther Gómez San Martín ${ }^{1} \quad$ Ana Gil Guerra ${ }^{1} \quad$ Israel Sanchez Lite ${ }^{1} \quad$ José Herreros Rodriguez ${ }^{2}$ \\ ${ }^{1}$ Servicio de Radiodiagnóstico, Hospital Clínico Universitario \\ Valladolid, Valladolid, España \\ 2 Servicio de Cirugía General y del Aparato Digestivo, Hospital Clínico \\ Universitario Valladolid, Valladolid, España \begin{abstract}
Radiologist, Medical Specialist, Department of Radiology, Hospital Clínico Universitario Valladolid, C/ Armuña 1, 4A, C.P. 47008, Valladolid, España (e-mail: hestergomez17@hotmail.com).
\end{abstract} \\ Address for correspondence Maria Esther Gómez San Martín,
}

Urol Colomb 2019;28:39-42.

\section{Resumen \\ Palabras Clave \\ - Carcinoma Uraco \\ - neoplasia \\ - uraco \\ - tomografía computarizada \\ - PET-TC}

Objetivo Revisar la patología tumoral del uraco, haciendo énfasis en su clínica, los métodos diagnósticos empleados y el manejo terapéutico.

Métodos Presentamos el caso de un varón de 39 años con carcinoma del uraco que simuló clínicamente un absceso umbilical.

Resultados Los hallazgos en la TC fueron sospechosos de patología del uraco. El examen anatomopatológico reveló células atípicas y la PET-TC demostró extensión a pared abdominal y epiplón, requiriéndose la resección completa.

Conclusiones El carcinoma del uraco es una neoplasia muy poco frecuente, que tiene su origen en el epitelio que recubre la luz del uraco, un vestigio de la alantoides que conecta la vejiga con el ombligo y normalmente involuciona en la etapa embrionaria. La clínica insidiosa hace que el diagnóstico sea tardío y el pronóstico pobre. Las pruebas de imagen tienen un papel fundamental en su sospecha, así como en la definición de la relación con la pared vesical y posible extensión local o a distancia. Una vez confirmado el diagnóstico histológicamente, está indicada la resección quirúrgica completa.

Objective To review tumoral pathology of urachus, emphasizing its clinical manifestations, diagnostic methods and therapeutic management.

Method We present the case of a 39-year-old male with urachal carcinoma who clinically mimicked an umbilical abscess.

Result CT findings were suspected of urachus pathology. Histologic analysis of the resected specimen demonstrated atypical cells, and PET-CT showed extension to the abdominal wall and omentum, requiring complete resection.

Conclusions Urachal carcinoma is a very rare neoplasm that originates in the epithelium that covers the lumen of the urachus, a vestige of the allantois that connects the bladder with the navel and normally involves in the embryonic stage. The insidious clinic makes late diagnosis and poor prognosis. Imaging tests play a fundamental role in their suspicion, as well as in the definition of the relationship with the bladder wall and possible local or distant extension. Once the diagnosis has been confirmed histologically, complete surgical resection is indicated. received

February 23, 2017

accepted

July 19, 2017

published online

May 2, 2018
DOI https://doi.org/

10.1055/s-0038-1648211.

ISSN 0120-789X.

eISSN 2027-0119.
Copyright ( 2019 , Sociedad Colombiana License terms de Urología. Publicado por Thieme Revinter Publicações Ltda., Rio de Janeiro, Brazil. Todos los derechos reservados.

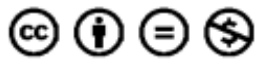




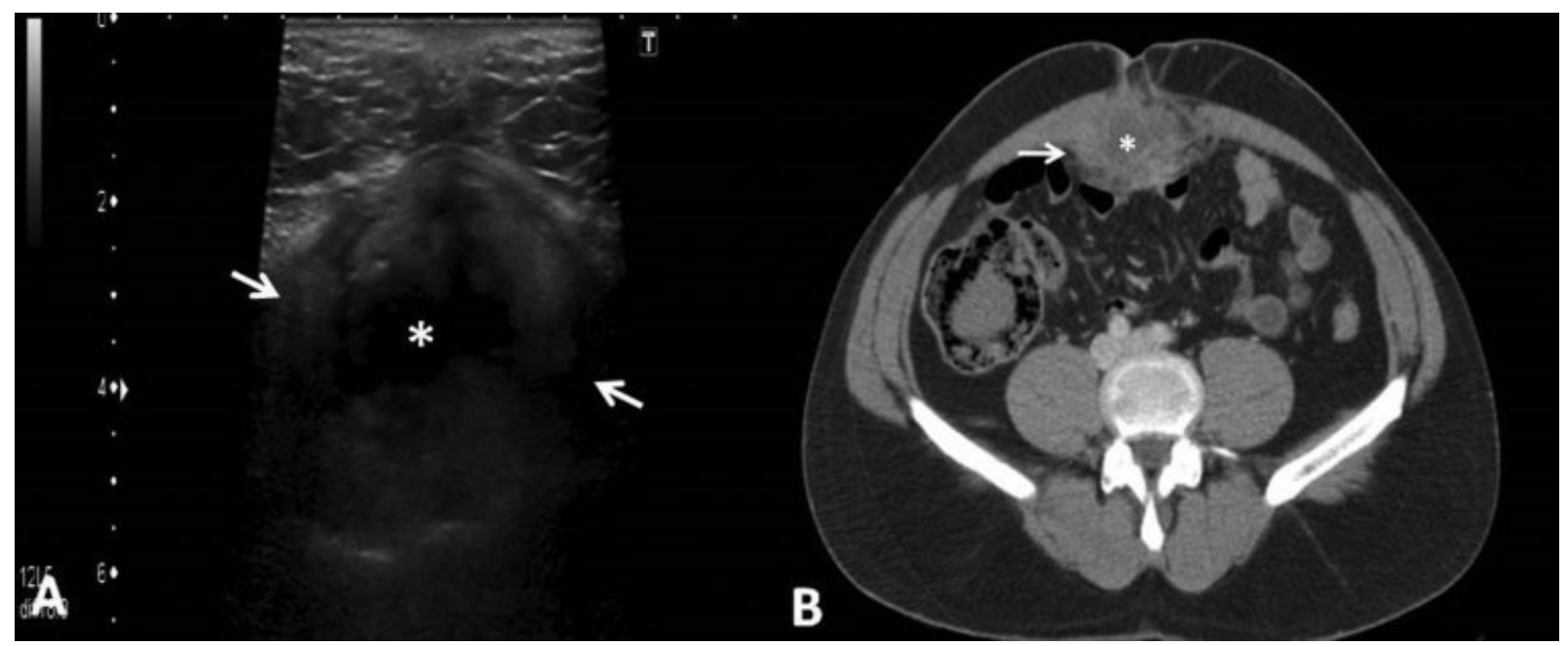

Fig. 1 (A) Ecografía transversal a nivel umbilical: lesión de ecogenicidad mixta con componente quístico-necrótico central, localizada profundamente a pared abdominal anterior. (B) TC abdominopélvico con contraste intravenoso: Lesión intraabdominal, con áreas quísticas y cambios inflamatorios entre los músculos rectos abdominales y el peritoneo.

\section{Introducción}

El uraco es un remanente embrionario de la alantoides, que comunica la vejiga con el ombligo y normalmente involuciona a una estructura fibromuscular en el tercer trimestre del embarazo. ${ }^{1,2}$ En un tercio de los adultos, se puede encontrar un remanente del uraco por fallo en el cierre completo de su luz. ${ }^{1}$ El carcinoma del uraco es extremadamente raro, representando entre el 0 y el $0,3 \%$ de los tumores vesicales y menos del $0,01 \%$ de todos los cánceres. El 90\% de los casos son adenocarcinomas. ${ }^{2,3}$

El diagnóstico clínico resulta difícil, pues requiere el uso de imagen y examen anatomopatológico para su confirmación. La combinación de tomografía por emisión de positrones (PET) con tomografía computarizada (TC) mejora la precisión en la estadificación prequirúrgica. ${ }^{4}$

El propósito de este trabajo es revisar la patología tumoral del uraco, haciendo hincapié en sus manifestaciones clínicas, reconocer el papel que desempeñan las distintas técnicas de imagen en su diagnóstico, y el manejo terapéutico.

\section{Material y Métodos}

Varón de 39 años, sin antecedentes de interés, que acude a Urgencias por cuadro clínico de dolor abdominal, focalizado en el ombligo, de un año de evolución y que se ha intensificado en el último mes. En la exploración presenta dolor a la palpación y sensación de masa a nivel umbilical. En la analítica se registran leucocitosis y aumento de "PCR" (reactantes de fase aguda). Se solicita ecografía abdominal donde se visualiza, coincidiendo con la tumoración palpable, una lesión de ecogenicidad mixta intraabdominal, en íntima relación con la pared abdominal, con contenido líquido y cambios inflamatorios locales; se completa estudio con TC abdominopélvico con contraste intravenoso que confirma la existencia de una lesión heterogénea, con componentes quísticos y extensos cambios inflamatorios, localizada a nivel umbilical con extensión longitudinal hacia la pelvis (-Fig. 1).

Con el diagnóstico de absceso umbilical y la posibilidad de patología relacionada con la persistencia del uraco, se decide tratamiento quirúrgico urgente. El hallazgo intraoperatorio es un absceso periumbilical con plastrón epiploico. Se realiza examen histológico de la muestra de peritoneo, evidenciándose intensa reacción inflamatoria aguda abscesificada, así como la presencia de algunas células atípicas positivas para marcadores Gata3 y CKAE1AE3, compatibles con el origen urotelial o en relación con restos embrionarios del uraco (-Fig. 2). Ante dichos resultados, se realiza un estudio PET-TC, donde se visualiza foco hipermetabólico en región retroumbilical (SUVmax $=12,2)$, que sugiere un área de inflamación $\mathrm{y}$ malignidad en músculo recto abdominal y epiplón (-Fig. 3).

Se decide realizar cirugía oncológica con la práctica de cistoscopia preoperatoria que resulta normal, por lo que se descarta la cistectomía. Se lleva a cabo una exéresis radical en bloque de pared abdominal incluyendo plano muscular, peritoneo y epiplón adherido y se reseca distalmente el uraco hasta la entrada a la vejiga.

\section{Conclusiones}

Los carcinomas uracales son tumores muy infrecuentes, constituyendo entre el 0 , y el $0,3 \%$ de los carcinomas vesicales, ${ }^{3,5}$ y plantean un importante reto diagnóstico ya que pueden simular otras entidades.

El uraco es un vestigio de dos estructuras embriológicas: la cloaca, que es la extensión cefálica del seno urogenital (precursor de la vejiga fetal) y la alantoides, que deriva del saco de Yolk.

Suele involucionar antes del nacimiento, hacia el quinto mes de gestación, y queda como remanente un cordón fibroso (ligamento umbilical mediano), una estructura 

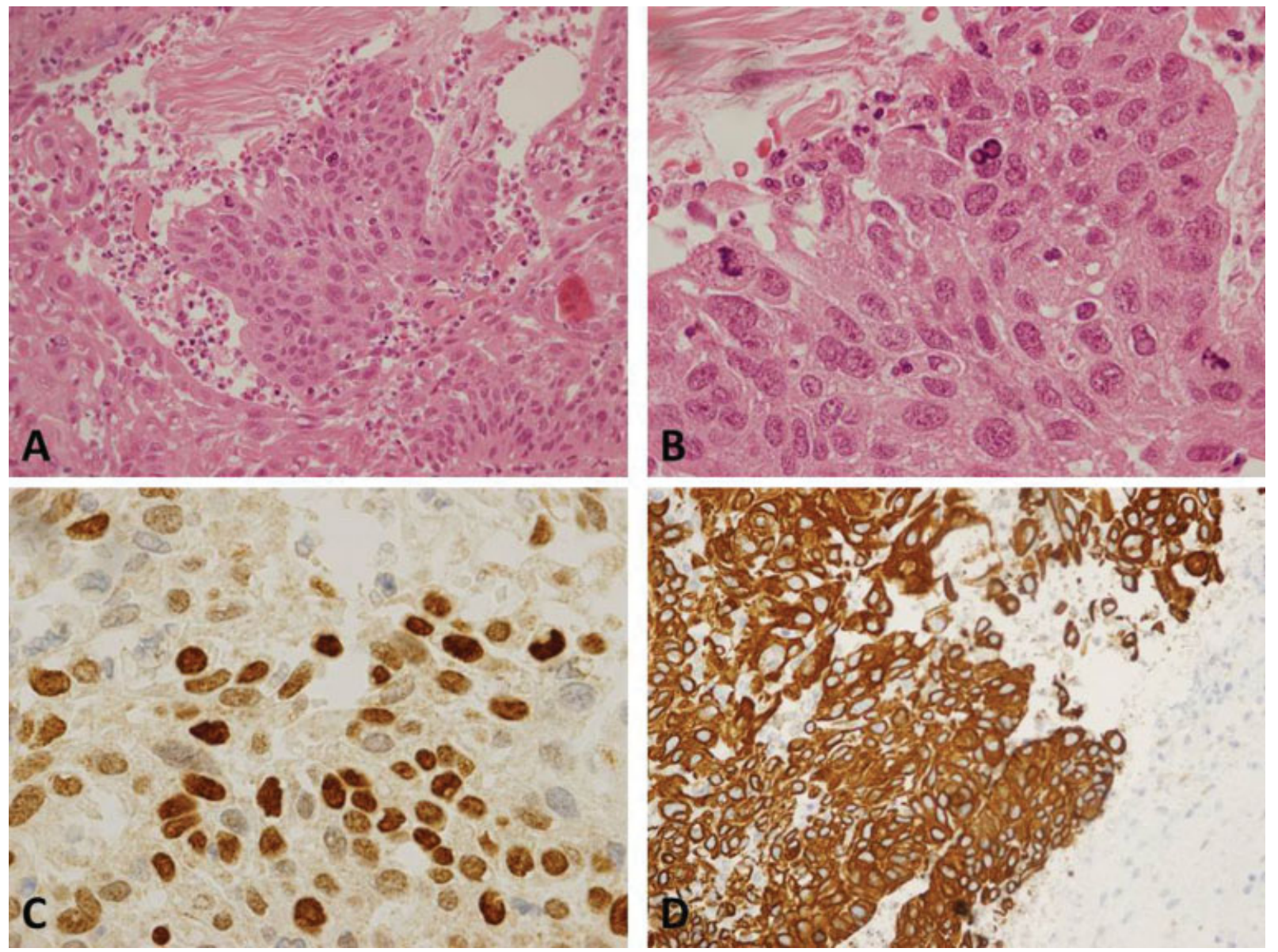

Fig. 2 Tejido de epiplón y peritoneo parietal. (A y B) Tinción: Hematoxilina-eosina. Aumento 40x y 100x. Se objetiva inflamación crónica linfoplasmocitaria y un grupo de células atípicas de aspecto tumoral, de tamaño grande, aumento del número de mitosis y alta relación núcleocitoplasma. Las células atípicas con técnicas de inmunohistoquímica son positivas para GATA3 (C) y CKAE1-AE3 (D), fenotipo tumoral compatible con origen urotelial o restos embrionarios del uraco.

tubular que transcurre en la línea media de la pared abdominal anterior entre el ápex de la vejiga urinaria y el ombligo, localizada en el espacio de Retzius entre la fascia transversal anteriormente y el peritoneo posteriormente y por tanto de localización extraperitoneal. ${ }^{1,2}$

Hay cuatro tipos de anomalías uracales congénitas ${ }^{1}$ : quiste uracal (una colección del uraco cerrada tanto en su extremo craneal como caudal, que suele localizarse en el tercio inferior del uraco), uraco persistente (cuando existe comunicación entre la luz vesical y el ombligo), seno uracal (persistencia del uraco en su extremo umbilical, estando el extremo vesical obliterado) y divertículo uracal (persistencia del uraco sólo en el extremo vesical).

La mayoría de pacientes con patología del uraco (salvo el uraco persistente) están asintomáticos. Sin embargo, se vuelven sintomáticos si se asocian con infección y otras complicaciones como neoplasias. ${ }^{2,6}$

Las neoplasias benignas que afectan al uraco incluyen adenomas, fibromas, fibroadenomas, fibromiomas y hamartomas, y son sumamente raros pudiendo simular lesiones malignas.

El uraco contiene una luz virtual recubierta por epitelio transicional, la metaplasia de dicho epitelio sería un paso previo necesario al desarrollo de una neoplasia, seguido de la transformación maligna. La mayoría de las neoplasias malignas son del subtipo adenocarcinoma mucosecretor (90\%) y representan aproximadamente un tercio de los adenocarcinomas que afectan a la vejiga urinaria.

Los carcinomas del uraco tienen predilección masculina, lo más frecuente es su aparición en varones entre 40 y 70 años.

Los síntomas de presentación más frecuentes son hematuria (73\%), dolor abdominal (14\%) y mucosuria a través del ombligo (10\%). ${ }^{2,5,7}$

Las distintas técnicas de imagen (ecografía, TC, resonancia magnética $)^{4,8}$ son de gran utilidad para detectar la lesión y definir su relación con la pared vesical, así como valorar la extensión a estructuras adyacentes (especialmente la grasa prevesical y la pared abdominal) y evaluar los posibles signos de diseminación a distancia. La lesión se presenta como una tumoración solitaria que invade la pared de la vejiga y con un componente predominantemente extravesical, situado en la línea media por delante y por encima de la vejiga en dirección al ombligo.

El tumor tiende a invadir la grasa supravesical y las estructuras de la pared abdominal. A causa de su 

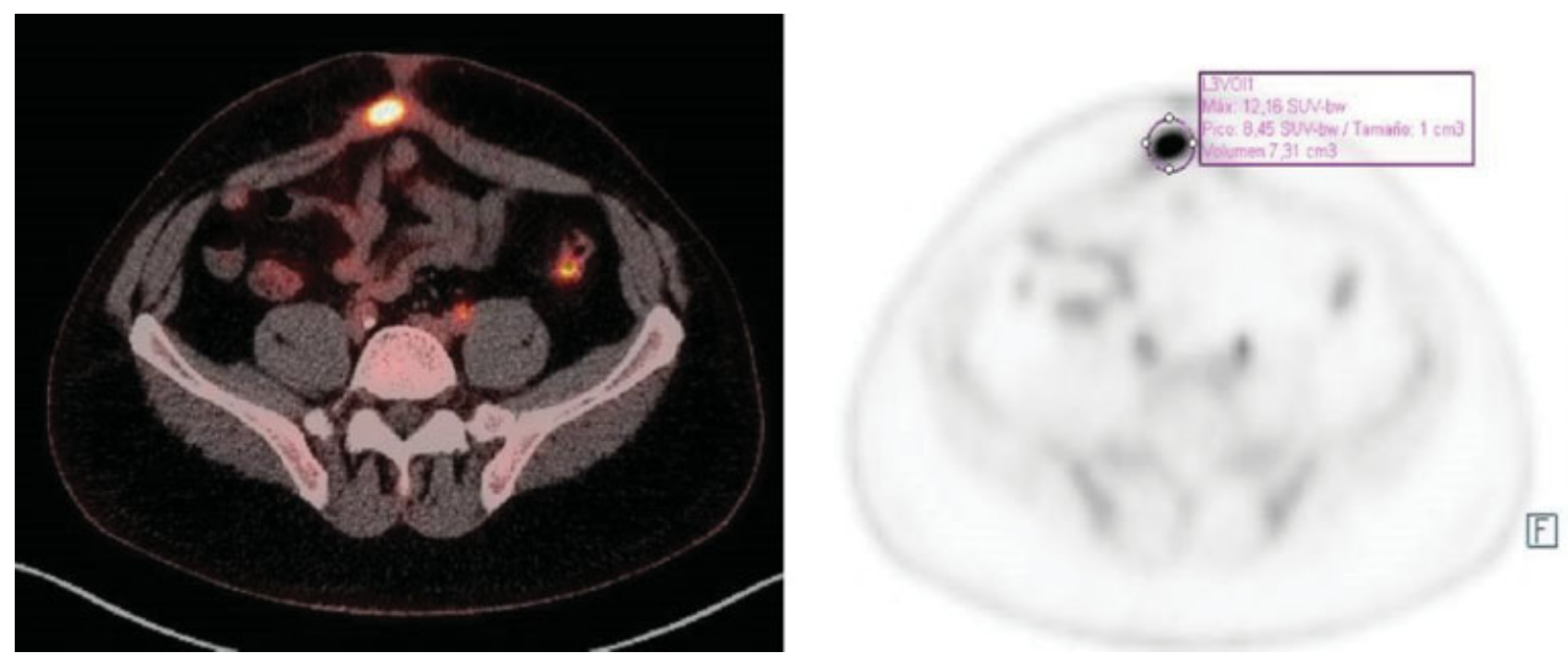

Fig. 3 PET-TC con 9,1 mCi de 18FDG: foco hipermetabólico retroumbilical (SUVmax =12,2 y 1,5 × 3,1 cm APxT), que se corresponde con lesión de partes blandas, sugestiva de área de inflamación y malignidad en músculo recto y epiplón.

localización extraperitoneal, los carcinomas del uraco son típicamente silentes y por consiguiente, la mayoría de pacientes muestran invasión local o enfermedad metastásica (ganglios linfáticos pélvicos, pulmón, cerebro, hígado o hueso) en el momento del diagnóstico.

Es frecuente encontrar calcificaciones psamomatosas periféricas hasta en el $50-70 \%$ de casos, que pueden ser de morfología punteada o curvilínea. Debido a que el tumor produce mucina, hasta en un $75 \%$ de los pacientes, puede llegar a dar una apariencia quística. ${ }^{7}$

Muchas veces es difícil distinguir entre un remanente del uraco infectado de un carcinoma del uraco. La presencia de hematuria, la detección en las técnicas de imagen de nódulos murales y calcificaciones, así como la ausencia de cambios inflamatorios locorregionales puede ser útil para su distinción en algunos casos. ${ }^{8}$

La cirugía es el tratamiento de elección. El pronóstico de los tumores uracales depende del estadio y del grado de diferenciación. Suele ser pobre debido a la escasa clínica que producen, el diagnóstico tardío, las recurrencias locales y metástasis a distancia. ${ }^{9,10}$ Uno de los principales factores de riesgo es la positividad de los márgenes quirúrgicos, por lo que es necesario una exéresis en bloque con extirpación completa del remanente uraco y ombligo.

\section{Responsabilidades Éticas}

Protección de personas y animales. Los autores declaran que para esta investigación no se han realizado experimentos en seres humanos ni en animales.

Confidencialidad de los datos. Los autores declaran que han seguido los protocolos de su centro de trabajo sobre la publicación de datos de pacientes.
Derecho a la privacidad y consentimiento informado. Los autores declaran que en este artículo no aparecen datos de pacientes.

\section{Conflicto de Intereses}

Los autores declaran no tener ningún tipo de conflicto de intereses.

\section{Bibliografía}

1 Upadhyay V, Kukkady A. Urachal remnants: an enigma. Eur J Pediatr Surg 2003;13(06):372-376

2 Ashley RA, Inman BA, Sebo TJ, et al. Urachal carcinoma: clinicopathologic features and long-term outcomes of an aggressive malignancy. Cancer 2006;107(04):712-720

3 Koster IM, Cleyndert P, Giard RW. Best cases from the AFIP: urachal carcinoma. Radiographics 2009;29(03):939-942

4 Yu JS, Kim KW, Lee HJ, Lee YJ, Yoon CS, Kim MJ. Urachal remnant diseases: spectrum of CT and US findings. Radiographics 2001;21 (02):451-461

5 Dhillon J, Liang Y, Kamat AM, et al. Urachal carcinoma: a pathologic and clinical study of 46 cases. Hum Pathol 2015;46 (12):1808-1814

6 Mengiardi B, Wiesner W, Stoffel F, Terracciano L, Freitag P. Case 44: Adenocarcinoma of the urachus. Radiology 2002;222(03):744-747

7 Mangiacapra FJ, Scheraga JL, Jones LA. Mucinous colloid adenocarcinoma of the urachus. Radiographics 2001;21(04):965-969

8 Wong-You-Cheong JJ, Woodward PJ, Manning MA, Sesterhenn IA. From the Archives of the AFIP: neoplasms of the urinary bladder: radiologic-pathologic correlation. Radiographics 2006;26(02): 553-580

9 Wright JL, Porter MP, Li CI, Lange PH, Lin DW. Differences in survival among patients with urachal and nonurachal adenocarcinomas of the bladder. Cancer 2006;107(04):721-728

10 Szarvas T, Módos O, Niedworok C, et al. Clinical, prognostic, and therapeutic aspects of urachal carcinoma-A comprehensive review with meta-analysis of 1,010 cases. Urol Oncol 2016;34(09):388-398 